\title{
APLICAÇÃO SEQÜENCIAL DE CLORETO DE MEPIQUAT EM ALGODOEIRO1
}

\author{
MANOEL LUIZ FERREIRA ATHAYDE² e FERNANDO MENDES LAMAS 3
}

\begin{abstract}
RESUMO - Com o objetivo de avaliar o efeito de doses de cloreto de mepiquat aplicadas de forma parcelada, foi conduzido, em Jaboticabal, SP, um experimento com a cultivar de algodoeiro IAC 22. $\mathrm{O}$ delineamento experimental foi o de blocos casualizados, com 13 tratamentos e 4 repetições. $\mathrm{O}$ efeito do cloreto de mepiquat sobre a redução da altura das plantas foi mais evidenciado pela dose total aplicada do que pelo uso do esquema de parcelamento. A menor dose estudada ( $55 \mathrm{~g} / \mathrm{ha})$ foi suficiente para que as plantas, por ocasião da colheita, estivessem com altura inferior a 1,30 m. O cloreto de mepiquat proporcionou redução no comprimento dos ramos e um melhor equilíbrio entre as partes reprodutiva e vegetativa. As características peso de capulho, peso de 100 sementes, porcentagem de fibra e produção de algodão em caroço, não foram significativamente afetadas pelos tratamentos.
\end{abstract}

Termos para indexação: Gossypium hirsutum, regulador de crescimento.

\section{SEQUENTIAL APPLICATIONS OF MEPIQUAT CHLORIDE IN COTTON PLANTS}

\begin{abstract}
The objective of this work was to evaluate the effect of split dosis of mepiquat chloride on the cotton cultivar IAC 22 at Jaboticabal, SP. The experimental design was in completely randomized blocks constituted by 13 treatments, and four replicates. The effect of dosis on plant height was more pronounced than the splitting effect. The smallest dosis used $(55 \mathrm{~g} / \mathrm{ha})$ was enough to declare plant height to less than $1.30 \mathrm{~m}$, at harvest. The effect of mepiquat chloride reduced branch length and provided better reproductive/vegetative relation. The effects on boll weight, 100 seed weight, percentage of fiber and seed yield were not significant.
\end{abstract}

Index terms: Gossypium hirsutum, growth regulator, cotton seed.

\section{INTRODUÇÃO}

A manipulação da arquitetura do dossel das plantas com biorreguladores é uma das mais recentes estratégias agronômicas para o incremento da produção de algodão (Hodges et al., 1991). O uso destes produtos em algodoeiro, principalmente quando cultivado em solos de alta fertilidade, vem aumentando, especialmente em propriedades onde se emprega a colheita mecanizada (Carvalho et al., 1994).

\footnotetext{
${ }^{1}$ Aceito para publicação em 12 de agosto de 1998.

${ }^{2}$ Eng. Agr., Dr., Prof. Titular, Dep. de Fitotecnia, Faculdade de Ciências Agrárias e Veterinárias de Jaboticabal (FCAVJ/UNESP), Rodovia Carlos Tonanni, Km 5, CEP 14870-000 Jaboticabal, SP.

${ }^{3}$ Eng. Agr., Dr., Embrapa-Centro de Pesquisa Agropecuária do Oeste (CPAO), Caixa Postal 661, CEP 79804-970 Dourados, MS. E-mail:lamas@cpao.embrapa.br
}

Resultados de pesquisas com o cloreto de mepiquat (cloreto 1,1- dimethyl piperidinum) permitem concluir que o produto pode auxiliar no estabelecimento de culturas mais lucrativas, uma vez que seus benefícios potenciais são: redução do crescimento vegetativo, melhoria da arquitetura das plantas, abertura precoce dos frutos, melhor eficiência na colheita mecanizada e superiores qualidades dos produtos finais (Barbosa \& Castro, 1983; Reddy et al., 1990).

Quando cultivado em condições onde não há limitações de umidade e a disponibilidade de nutrientes é adequada, o algodoeiro produz excessiva vegetação, que pode interferir negativamente na produção final; em tal situação, o uso de regulador de crescimento torna-se inevitável (Reddy et al., 1992). De acordo com Meredith Junior \& Wells (1989), o ideal é que a relação entre a parte reprodutiva e a vegetativa do algodoeiro seja maior que a unidade; neste caso, a correlação com a produção é positiva. 
Embora os efeitos do cloreto de mepiquat sobre o crescimento excessivo das plantas sejam devidos à redução da extensão do caule, menor número de nós, menor comprimento de ramos e decréscimo da área foliar (York, 1983; Stuart et al., 1984; Kerby, 1985; Reddy et al., 1990; Fernandez et al., 1991), as respostas sobre a produção de algodão são inconsistentes (Hodges et al., 1991).

Os efeitos do cloreto de mepiquat sobre o algodoeiro dependem de vários fatores, podendo-se destacar: temperatura, população de plantas, época de semeadura, cultivar, época de aplicação e dose utilizada (York, 1983; Reddy et al., 1990; Reddy et al., 1992; Wallace et al., 1993; Carvalho et al.,1994).

Com relação à dose utilizada, segundo Wallace et al. (1993), o parcelamento das aplicações de cloreto de mepiquat tem efeito mais marcante em relação à aplicação única sobre a altura de plantas, número de nós e comprimento dos internódios. A retenção de frutos também foi maior nas aplicações parceladas.

Nas cultivares que apresentaram excessivo crescimento vegetativo, os efeitos do cloreto de mepiquat foram benéficos; por outro lado, nas cultivares de porte baixo o efeito do produto não foi significativo (Cia et al.,1984).

Segundo Hodges et al. (1991), o efeito do cloreto de mepiquat é altamente dependente da temperatura, uma vez que o produto interfere em processos fisiológicos da planta, como fotossíntese e respiração.

O presente trabalho foi conduzido com o objetivo de avaliar os efeitos de diferentes doses de cloreto de mepiquat, aplicadas de forma parcelada, sobre algumas características do algodoeiro cultivar IAC 22 .

\section{MATERIAL E MÉTODOS}

O experimento foi conduzido no ano agrícola de 1995/96, em área da Fazenda Experimental da FCAVJ/ UNESP, localizada em Jaboticabal, SP, com coordenadas geográficas de $21^{\circ} 15^{\prime} 23^{\prime \prime}$ de latitude sul e $48^{\circ} 18^{\prime} 58^{\prime \prime}$ de longitude oeste, em um Latossolo Vermelho-Escuro eutrófico. Utilizou-se a cultivar IAC 22, semeada em 31/10/95, com emergência em $07 / 11 / 95$, com espaçamento entre fileiras de $0,90 \mathrm{~m}$ e 8 plantas por metro linear.

$\mathrm{O}$ delineamento experimental utilizado foi blocos casualizados, com 13 tratamentos e quatro repetições. Os tratamentos estudados se encontram na Tabela 1.

O produto comercial utilizado foi o PIX com $5 \%$ de cloreto de mepiquat $(50 \mathrm{~g} / \mathrm{L})$.

Por ocasião da primeira aplicação, as plantas apresentavam altura média de $62,35 \mathrm{~cm}$; na segunda $82,42 \mathrm{~cm}$ e na terceira $100,13 \mathrm{~cm}$.

Cada parcela experimental era constituída por quatro fileiras de plantas com espaços, entre si, de $0,90 \mathrm{~m}$, com $35,0 \mathrm{~m}$ de comprimento. Entre as parcelas, dentro do bloco, foi deixado um intervalo de $2,0 \mathrm{~m}$, e entre blocos, 4,0 m. As aplicações de cloreto de mepiquat foram rea-

TABELA 1. Dose (g/ha) total aplicada de cloreto de mepiquat e seu parcelamento em 3 aplicações, aos 50, 64 e 78 dias após a emergência (DAE) do algodoeiro cv. IAC 22. Jaboticabal, SP, 1996.

\begin{tabular}{ccccc}
\hline Tratamento & $\begin{array}{c}1^{\text {a }} \text { aplicação } \\
(50 \text { DAE })\end{array}$ & $\begin{array}{c}2^{\text {a }} \text { aplicação } \\
(64 \text { DAE })\end{array}$ & $\begin{array}{c}3^{\text {a }} \text { aplicação } \\
(78 \text { DAE })\end{array}$ \\
\hline 1 & 15 & 25 & 15 & 55 \\
2 & 15 & 25 & 25 & 65 \\
3 & 15 & 35 & 15 & 65 \\
4 & 15 & 35 & 25 & 75 \\
5 & 25 & 25 & 15 & 75 \\
6 & 25 & 25 & 25 & 75 \\
7 & 25 & 35 & 25 & 85 \\
8 & 25 & 35 & 15 & 75 \\
1 & 35 & 25 & 25 & 85 \\
1 & 35 & 25 & 15 & 85 \\
13 & 35 & 35 & 25 & 0 \\
\end{tabular}


lizadas com pulverizador de barras, equipado com bicos cônicos, com uma vazão de 270 litros de calda/ha, tracionado por trator.

O controle de plantas daninhas foi realizado com a aplicação em pré-plantio incorporado, do herbicida trifluralin, utilizando-se $2,00 \mathrm{~L} /$ ha de produto comercial com $445 \mathrm{~g} / \mathrm{L}$ do i.a., dois cultivos mecânicos e uma capina manual. A adubação de semeadura foi realizada de acordo com as recomendações para a cultura no Estado de São Paulo, aplicando-se $12 \mathrm{~kg} / \mathrm{ha}$ de N, $60 \mathrm{~kg} / \mathrm{ha}$ de $\mathrm{K}_{2} \mathrm{O}$ e $60 \mathrm{~kg} / \mathrm{ha}$ de $\mathrm{P}_{2} \mathrm{O}_{5}$. A primeira adubação de cobertura foi realizada imediatamente após o desbaste, com $40 \mathrm{~kg} / \mathrm{ha}$ de $\mathrm{N}$ na forma de sulfato de amônia, mais $39 \mathrm{~kg} / \mathrm{ha}$ de $\mathrm{K}_{2} \mathrm{O}$ na forma de cloreto de potássio, e $0,8 \mathrm{~kg}$ de boro na forma de bórax. Aos trinta e cinco dias após a emergência realizou-se a segunda adubação em cobertura com $40 \mathrm{~kg} / \mathrm{ha}$ de $\mathrm{N}$ na forma de sulfato de amônia. O controle de pragas foi realizado de acordo com Busoli et al. (1992).

A partir da primeira aplicação do cloreto de mepiquat, a cada sete dias, até aos $100 \mathrm{DAE}$, foi realizado o monitoramento da altura das plantas, medindo-se, de cada parcela experimental, a altura de dez plantas tomadas ao acaso.

Aos 30, 60, 90 e 100 DAE, coletaram-se em cada parcela experimental, cinco plantas, para as seguintes avaliações: matéria seca do caule, das folhas e dos frutos, e número de frutos. Aos 100 DAE determinou-se também a área foliar com equipamento "Portable Area Meter" Licor Model Li-3000 A .
Por ocasião da primeira colheita, realizada aos 140 DAE, na área útil de cada parcela $\left(9,0 \mathrm{~m}^{2}\right)$, utilizando-se dez plantas, foram feitas as seguintes determinações: número de capulhos, número de maçãs, matéria seca do caule e das folhas, comprimento dos ramos do quinto, sétimo, nono e décimo primeiro nó, e comprimento dos seis últimos internódios.

Imediatamente antes da primeira colheita, de acordo com metodologia proposta por Sabino et al. (1975), foi coletado o algodão em caroço de 30 capulhos, para se obter o seu peso médio. Após o descaroçamento das amostras, determinou-se o peso de 100 sementes e a porcentagem de fibra. $\mathrm{O}$ algodão em caroço obtido em cada parcela experimental foi pesado em balança de precisão, e o resultado, transformado em $\mathrm{kg} / \mathrm{ha}$.

Os resultados obtidos foram submetidos a análise de variância, e posteriormente foi aplicado o teste de Tukey, a 5\%, para comparação das médias dos tratamentos. Sempre que necessário, para a análise de variância, foi feita a transformação dos dados Banzatto \& Kronka (1992).

\section{RESULTADOS E DISCUSSÃO}

Nas avaliações realizadas aos 7, 14, 21 dias após a última aplicação (DAA) e por ocasião da colheita, o efeito do cloreto de mepiquat, para a variável altura de plantas foi significativo (Tabela 2). Nos quatro períodos em que foram feitas as avaliações, as

TABELA 2. Altura média das plantas (cm) aos 7, 14 e 21 dias após a aplicação (DAA) e na colheita do algodoeiro cv. IAC 22, submetido a tratamentos com cloreto de mepiquat. Jaboticabal, SP, 1996 ${ }^{\text {. }}$

\begin{tabular}{ccccc}
\hline Tratamento & \multicolumn{3}{c}{ Altura das plantas $(\mathrm{cm})$} & \multicolumn{1}{c}{ Colheita } \\
\cline { 2 - 5 } & 7 DAA & 14 DAA & $110,56 \mathrm{~b}$ & $109,75 \mathrm{bc}$ \\
1 & $105,00 \mathrm{~b}$ & $107,42 \mathrm{~b}$ & $107,19 \mathrm{bcde}$ & $108,06 \mathrm{~cd}$ \\
3 & $104,63 \mathrm{~b}$ & $103,19 \mathrm{de}$ & $105,75 \mathrm{cdef}$ & $113,19 \mathrm{~b}$ \\
4 & $105,37 \mathrm{~b}$ & $107,06 \mathrm{~b}$ & $107,19 \mathrm{bcde}$ & $107,94 \mathrm{~cd}$ \\
5 & $102,56 \mathrm{~b}$ & $107,62 \mathrm{~b}$ & $108,06 \mathrm{bcd}$ & $109,62 \mathrm{bc}$ \\
6 & $102,25 \mathrm{~b}$ & $104,00 \mathrm{cde}$ & $107,25 \mathrm{bcde}$ & $106,81 \mathrm{cde}$ \\
7 & $105,70 \mathrm{~b}$ & $106,33 \mathrm{bc}$ & $104,12 \mathrm{f}$ & $104,69 \mathrm{def}$ \\
8 & $102,75 \mathrm{~b}$ & $101,44 \mathrm{e}$ & $106,37 \mathrm{cdef}$ & $106,44 \mathrm{cde}$ \\
9 & $101,12 \mathrm{~b}$ & $101,12 \mathrm{e}$ & $109,37 \mathrm{bc}$ & $110,81 \mathrm{bc}$ \\
10 & $103,37 \mathrm{~b}$ & $105,06 \mathrm{bcd}$ & $105,50 \mathrm{def}$ & $107,81 \mathrm{~cd}$ \\
11 & $101,37 \mathrm{~b}$ & $103,37 \mathrm{cde}$ & $104,94 \mathrm{ef}$ & $102,50 \mathrm{ef}$ \\
12 & $98,62 \mathrm{~b}$ & $98,01 \mathrm{f}$ & $103,38 \mathrm{f}$ & $101,75 \mathrm{f}$ \\
13 & $103,02 \mathrm{~b}$ & $103,02 \mathrm{de}$ & $135,62 \mathrm{a}$ & $136,31 \mathrm{a}$ \\
\hline CV $(\%)$ & $126,18 \mathrm{a}$ & $132,37 \mathrm{a}$ & 4,01 & 4,86 \\
\hline
\end{tabular}

${ }^{1}$ Médias de tratamentos, seguidas pela mesma letra, não diferem entre si, pelo teste de Tukey, a $5 \%$ de probabilidade. 
plantas do tratamento-testemunha foram as que apresentaram a maior altura. Aos 14 DAA, a média do tratamento $(35+35+15=85 \mathrm{~g}$ i.a./ha) diferiu significativamente da média dos demais tratamentos, apresentando o menor valor. Aos 21 DAA, os tratamentos 3, 7, 8, 10, 11 e 12 apresentaram as menores médias, que não diferiram entre si, pelo teste de Tukey a 5\%. A altura final, medida por ocasião da colheita, foi significativamente menor nos tratamentos 7, 11 e 12, não diferindo entre si. Considerando que, especialmente para a colheita mecânica o ideal é que as plantas tenham altura entre 1,20 a 1,30 m, a dose de $55 \mathrm{~g}$ i.a./ha foi suficiente, pois para este tratamento, quando da colheita, as plantas tinham altura de $109,75 \mathrm{~cm}$. O efeito do cloreto de mepiquat como regulador do crescimento das plantas em altura foi constatado também por York (1983), Stuart et al. (1984), Reddy et al. (1990) e Carvalho et al. (1994).

No presente trabalho, quando se analisa o efeito do cloreto de mepiquat aplicado na mesma dose total, mas parcelado de forma diferente no tocante à altura das plantas, não se verificaram diferenças significativas. Verificou-se apenas certa tendência das plantas que receberam a menor dose na última aplicação, de apresentarem maior altura. Este comportamento indica que, quando do parcelamento, a última aplicação deve ser feita com uma dose no mínimo igual às anteriores. Wallace et al. (1993) relatam que o efeito do cloreto de mepiquat quando aplicado parcelado, depende do esquema de parcelamento adotado.

A matéria seca do caule, avaliada aos 100 dias após a emergência, foi significativamente influenciada pelo cloreto de mepiquat (Tabela 3), graças ao qual o tratamento-testemunha apresentou o maior valor. A média do tratamento 12 (95 g i.a./ha) foi significativamente menor que as médias dos tratamentos 5, 6 , 7, 8 e $9(65,75,75,85$ e 75 g i.a./ha).

$\mathrm{O}$ efeito do cloreto de mepiquat sobre a matéria seca foliar avaliada aos 100 dias após a emergência não ficou bem evidenciado (Tabela 3). Não se verificou, pelo teste de Tukey a 5\%, diferença significativa entre o tratamento-testemunha (13) e o tratamento que recebeu a maior dose, entre as doses estudadas (95 g/ha). Stuart et al. (1984), trabalhando com a dose de $50 \mathrm{~g}$ de cloreto de mepiquat/ha, veri- ficaram que o produto provocou redução no tamanho das folhas. Entretanto, os autores não comentam sobre o efeito do cloreto de mepiquat sobre a matéria seca foliar.

Os tratamentos 6,10,11 e 13 apresentaram a maior área foliar; entretanto, não se verificou diferença significativa, entre eles, pelo teste de Tukey (Tabela 3). Barbosa \& Castro (1983), Reddy et al. (1990) e Fernandes et al. (1991) relatam que o cloreto de mepiquat provoca redução da área foliar, o que no presente trabalho não ficou evidenciado. Entre as doses de cloreto de mepiquat, os tratamentos 1, 7 e 9 (55, 75 e $75 \mathrm{~g}$ i.a./ha) apresentaram os menores valores de área foliar sem haver entre eles diferença significativa. Pelos resultados obtidos, fica evidente que a área foliar medida aos 100 dias após a emergência foi mais influenciada pelas duas primeiras aplicações. Assim, para uma mesma dose total, os tratamentos em que as duas primeiras aplicações foram com as maiores doses, apresentaram menor área foliar.

A relação entre a matéria seca da parte reprodutiva e vegetativa foi significativamente influenciada pelos tratamentos (Tabela 3). Na maioria dos tratamentos que receberam cloreto de mepiquat, o valor da relação foi significativamente maior do que o tratamento-testemunha. Meredith Junior \& Wells (1989) citam que o ideal é que a relação entre a matéria seca da parte reprodutiva e vegetativa seja maior que a unidade, e quanto maior este valor, mais eficiente é a planta. Os autores acrescentam, ainda, que este valor normalmente se correlaciona positivamente com a produção de algodão em caroço.

As plantas tratadas com cloreto de mepiquat tiveram o comprimento dos ramos do quinto, sétimo, nono e décimo primeiro nó reduzido, o que é mais evidente nos ramos localizados no sétimo, nono e décimo primeiro nó (Tabela 4). Pelos resultados obtidos, fica bem evidenciado que o efeito do cloreto de mepiquat é maior nas partes da planta de ativo crescimento. Este efeito pode ser considerado como vantajoso, pois com a redução do comprimento dos ramos, têm-se maior retenção de frutos nas primeiras posições dos ramos. De acordo com Beltrão \& Azevêdo (1993), os frutos das primeiras posições dos ramos frutíferos são responsáveis por mais de $80 \%$ da produção total. O menor comprimento dos 
ramos é desejável também por facilitar a colheita, tanto manual como mecânica (Cruz et al., 1982).

O cloreto de mepiquat provocou redução significativa no comprimento dos seis últimos internódios (Tabela 4). Entre as doses estudadas, não foi possí- vel a discriminação da dose que provocou maior redução.

O tratamento $11(35+35+15 \mathrm{~g}$ i.a./ha) foi o que apresentou o menor valor de matéria seca foliar quando da colheita, sem, no entanto, diferir signi-

TABELA 3. Matéria seca (g) de caule e de folhas, área foliar $\left(\mathrm{cm}^{2}\right)$ e razão entre a matéria seca da parte reprodutiva e vegetativa, aos 100 dias após a emergência do algodoeiro cv. IAC 22, submetido a tratamentos com cloreto e mepiquat. Jaboticabal, SP, 1996 ${ }^{1}$.

\begin{tabular}{cllcc}
\hline Tratamento & MS caule & MS folhas & Área foliar & MSR/MSV \\
\hline 1 & $34,60 \mathrm{bcd}$ & $15,20 \mathrm{e}$ & $1776,07 \mathrm{f}$ & $1,30 \mathrm{bcd}$ \\
2 & $32,44 \mathrm{~d}$ & $17,66 \mathrm{cde}$ & $2257,61 \mathrm{de}$ & $1,31 \mathrm{abcd}$ \\
3 & $34,15 \mathrm{bcd}$ & $17,57 \mathrm{cde}$ & $2261,42 \mathrm{de}$ & $1,22 \mathrm{~cd}$ \\
4 & $33,61 \mathrm{~cd}$ & $18,34 \mathrm{~cd}$ & $2300,01 \mathrm{cde}$ & $1,22 \mathrm{~cd}$ \\
5 & $38,48 \mathrm{abc}$ & $16,72 \mathrm{de}$ & $2272,65 \mathrm{de}$ & $1,39 \mathrm{ab}$ \\
6 & $39,04 \mathrm{ab}$ & $22,34 \mathrm{a}$ & $2683,91 \mathrm{ab}$ & $1,18 \mathrm{de}$ \\
7 & $37,88 \mathrm{abc}$ & $17,21 \mathrm{cde}$ & $1980,14 \mathrm{ef}$ & $1,45 \mathrm{a}$ \\
8 & $38,32 \mathrm{abc}$ & $19,55 \mathrm{bc}$ & $2377,38 \mathrm{bcd}$ & $1,28 \mathrm{bcd}$ \\
9 & $37,99 \mathrm{abc}$ & $21,82 \mathrm{ab}$ & $1893,41 \mathrm{f}$ & $1,20 \mathrm{~cd}$ \\
10 & $35,39 \mathrm{bcd}$ & $19,13 \mathrm{~cd}$ & $2616,70 \mathrm{abc}$ & $1,38 \mathrm{ab}$ \\
11 & $36,93 \mathrm{abcd}$ & $22,42 \mathrm{a}$ & $2858,99 \mathrm{a}$ & $1,34 \mathrm{ab}$ \\
12 & $32,57 \mathrm{~d}$ & $18,92 \mathrm{~cd}$ & $2374,77 \mathrm{bcd}$ & $1,41 \mathrm{ab}$ \\
13 & $40,73 \mathrm{a}$ & $16,75 \mathrm{de}$ & $2784,96 \mathrm{a}$ & $1,05 \mathrm{e}$ \\
\hline CV $(\%)$ & 16,50 & 17,25 & 17,26 & 13,86 \\
\hline
\end{tabular}

${ }_{1}^{1}$ Médias de tratamentos, seguidas pela mesma letra, não diferem entre si, pelo teste de Tukey, a $5 \%$ de probabilidade.

TABELA 4. Comprimento de ramos $(\mathrm{cm})$ do quinto, sétimo, nono e décimo primeiro nó e comprimento médio dos seis últimos internódios $(\mathrm{cm})$ do algodoeiro cv. IAC 22, submetido a tratamentos com cloreto de mepiquat. Jaboticabal, SP, 1996'.

\begin{tabular}{clllcc}
\hline Tratamento & $\begin{array}{c}\text { Ramos do } \\
\text { quinto nó }\end{array}$ & $\begin{array}{c}\text { Ramos do } \\
\text { sétimo nó }\end{array}$ & $\begin{array}{c}\text { Ramos do } \\
\text { nono nó }\end{array}$ & $\begin{array}{c}\text { Ramos do décimo } \\
\text { primeiro nó }\end{array}$ & $\begin{array}{c}\text { Comprimento dos seis } \\
\text { últimos internódios }\end{array}$ \\
\hline 1 & $32,50 \mathrm{e}$ & $28,95 \mathrm{~g}$ & $24,37 \mathrm{~d}$ & $18,15 \mathrm{f}$ & $20,54 \mathrm{~b}$ \\
2 & $42,30 \mathrm{abc}$ & $36,82 \mathrm{~b}$ & $29,32 \mathrm{bc}$ & $23,27 \mathrm{bcd}$ & $19,22 \mathrm{bc}$ \\
3 & $41,0 \mathrm{bcd}$ & $34,42 \mathrm{bcde}$ & $30,30 \mathrm{bc}$ & $24,35 \mathrm{bc}$ & $19,13 \mathrm{bc}$ \\
4 & $35,75 \mathrm{de}$ & $35,50 \mathrm{bcd}$ & $26,30 \mathrm{~cd}$ & $19,07 \mathrm{ef}$ & $18,29 \mathrm{~cd}$ \\
5 & $33,32 \mathrm{e}$ & $32,25 \mathrm{defg}$ & $30,77 \mathrm{bc}$ & $20,77 \mathrm{def}$ & $19,84 \mathrm{bc}$ \\
6 & $46,15 \mathrm{ab}$ & $37,30 \mathrm{~b}$ & $31,42 \mathrm{~b}$ & $21,92 \mathrm{bcde}$ & $18,15 \mathrm{~d}$ \\
7 & $44,87 \mathrm{ab}$ & $33,00 \mathrm{cdef}$ & $29,90 \mathrm{bc}$ & $21,22 \mathrm{cdef}$ & $18,74 \mathrm{bcd}$ \\
8 & $42,70 \mathrm{abc}$ & $31,15 \mathrm{efg}$ & $29,20 \mathrm{bc}$ & $19,97 \mathrm{def}$ & $18,29 \mathrm{~cd}$ \\
9 & $38,55 \mathrm{cde}$ & $34,97 \mathrm{bcd}$ & $30,72 \mathrm{bc}$ & $25,30 \mathrm{~b}$ & $19,32 \mathrm{bc}$ \\
10 & $36,60 \mathrm{cde}$ & $30,50 \mathrm{fg}$ & $28,80 \mathrm{bcd}$ & $20,75 \mathrm{def}$ & $18,21 \mathrm{~cd}$ \\
11 & $42,80 \mathrm{abc}$ & $32,20 \mathrm{defg}$ & $26,75 \mathrm{~cd}$ & $21,57 \mathrm{cdef}$ & $18,99 \mathrm{bc}$ \\
12 & $41,65 \mathrm{bcd}$ & $35,35 \mathrm{bc}$ & $29,55 \mathrm{bc}$ & $21,15 \mathrm{cdef}$ & $19,16 \mathrm{bc}$ \\
13 & $47,90 \mathrm{a}$ & $40,67 \mathrm{a}$ & $38,95 \mathrm{a}$ & $30,90 \mathrm{a}$ & $22,31 \mathrm{a}$ \\
\hline $\mathrm{CV}(\%)$ & 18,32 & 13,14 & 18,27 & 19,62 & 12,14 \\
\hline
\end{tabular}

${ }^{1}$ Médias de tratamentos, seguidas pela mesma letra, não diferem entre si, pelo teste de Tukey, a 5\% de probabilidade. 
ficativamente da média dos tratamentos $1(15+25+15), 3(15+35+15), 5(25+25+15$ g i.a./ha), mas diferindo da testemunha. Os resultados obtidos no presente trabalho são semelhantes aos obtidos por Stuart et al. (1984) e Reddy et al. (1990). Quanto a isto, estes autores mencionam que o cloreto de mepiquat reduz a matéria seca foliar. Entretanto, os autores não mencionam o estádio fenológico, em que foram realizadas as avaliações.

A matéria seca do caule, avaliada quando da colheita, foi maior nos tratamentos 6,9 e 13, sem diferirem entre si, pelo teste de Tukey (Tabela 5).
$\mathrm{O}$ efeito do cloreto de mepiquat sobre o número de maçãs e de capulhos quando da colheita não ficou bem evidenciado (Tabela 5).

No tocante às características agronômicas peso de capulho, peso de 100 sementes, porcentagem de fibra e produção de algodão em caroço, o efeito do cloreto de mepiquat não foi significativo (Tabela 6).

Os resultados obtidos neste trabalho, no que diz respeito a todas as variáveis analisadas, concordam com relatos feitos por Cia et al. (1984), Reddy et al. (1990), Hodges et al. (1991), Reddy et al. (1992), Wallace et al. (1993) e Carvalho et al. (1994). Estes

TABELA 5. Matéria seca (g) de folhas, caule, número de maçãs e de capulhos por planta, por ocasião da colheita de algodoeiro cv. IAC 22, submetido a tratamentos com cloreto de mepiquat. Jaboticabal, SP, 1996 ${ }^{1}$.

\begin{tabular}{cllll}
\hline Tratamento & MS folhas & MS caule & No de maçãs $^{2}$ & $\mathrm{~N}^{\underline{0}}$ de capulhos \\
\hline 1 & $4,54 \mathrm{fg}$ & $29,82 \mathrm{~g}$ & $1,79 \mathrm{efg}$ & $7,64 \mathrm{cde}$ \\
2 & $5,99 \mathrm{def}$ & $34,50 \mathrm{def}$ & $2,07 \mathrm{a}$ & $8,15 \mathrm{abc}$ \\
3 & $5,83 \mathrm{defg}$ & $31,27 \mathrm{fg}$ & $1,66 \mathrm{~g}$ & $8,05 \mathrm{bcd}$ \\
4 & $6,50 \mathrm{~cd}$ & $35,44 \mathrm{cde}$ & $1,91 \mathrm{cde}$ & $7,50 \mathrm{cde}$ \\
5 & $4,93 \mathrm{efg}$ & $35,06 \mathrm{cde}$ & $1,75 \mathrm{fg}$ & $8,20 \mathrm{f}$ \\
6 & $8,02 \mathrm{ab}$ & $39,80 \mathrm{ab}$ & $1,92 \mathrm{bcde}$ & $7,75 \mathrm{ab}$ \\
7 & $7,24 \mathrm{bcd}$ & $37,67 \mathrm{bcd}$ & $2,09 \mathrm{a}$ & $7,70 \mathrm{cde}$ \\
8 & $7,17 \mathrm{bcd}$ & $33,84 \mathrm{ef}$ & $2,04 \mathrm{abc}$ & $5,73 \mathrm{f}$ \\
9 & $9,14 \mathrm{a}$ & $38,36 \mathrm{abc}$ & $2,06 \mathrm{ab}$ & $7,64 \mathrm{cde}$ \\
10 & $6,11 \mathrm{de}$ & $30,23 \mathrm{~g}$ & $2,00 \mathrm{abc}$ & $8,95 \mathrm{a}$ \\
11 & $4,45 \mathrm{~g}$ & $33,10 \mathrm{efg}$ & $1,76 \mathrm{fg}$ & $8,20 \mathrm{abc}$ \\
12 & $6,13 \mathrm{cde}$ & $34,33 \mathrm{def}$ & $1,71 \mathrm{~g}$ & $7,26 \mathrm{de}$ \\
\hline 13 & $7,60 \mathrm{bc}$ & $41,46 \mathrm{a}$ & $1,87 \mathrm{def}$ & 5,75 \\
\hline
\end{tabular}

${ }^{1}$ Médias de tratamentos, seguidas pela mesma letra, não diferem entre si, pelo teste de Tukey, a $5 \%$ de probablidade.

2 Dados transformados em $\sqrt{x+1}$.

TABELA 6. Peso médio (g) de capulho e de 100 sementes, porcentagem de fibra e produção de algodão em caroço (kg/ha) de algodoeiro cv. IAC 22, submetido a tratamentos com cloreto de mepiquat. Jaboticabal, SP, $1996^{1}$.

\begin{tabular}{ccccc}
\hline Tratamento & Capulho & Cem sementes & Fibra & Produção de algodão em caroço \\
\hline 1 & $7,26 \mathrm{a}$ & $13,05 \mathrm{a}$ & $34,43 \mathrm{a}$ & $3860,58 \mathrm{a}$ \\
2 & $7,41 \mathrm{a}$ & $11,98 \mathrm{a}$ & $35,14 \mathrm{a}$ & $3687,53 \mathrm{a}$ \\
3 & $7,65 \mathrm{a}$ & $12,60 \mathrm{a}$ & $35,24 \mathrm{a}$ & $3695,06 \mathrm{a}$ \\
4 & $7,44 \mathrm{a}$ & $11,97 \mathrm{a}$ & $34,93 \mathrm{a}$ & $3539,19 \mathrm{a}$ \\
5 & $7,28 \mathrm{a}$ & $12,20 \mathrm{a}$ & $35,29 \mathrm{a}$ & $3513,92 \mathrm{a}$ \\
6 & $7,73 \mathrm{a}$ & $12,62 \mathrm{a}$ & $34,74 \mathrm{a}$ & $3411,86 \mathrm{a}$ \\
7 & $7,30 \mathrm{a}$ & $11,85 \mathrm{a}$ & $35,24 \mathrm{a}$ & $3791,25 \mathrm{a}$ \\
8 & $7,49 \mathrm{a}$ & $12,40 \mathrm{a}$ & $3500,00 \mathrm{a}$ \\
9 & $7,57 \mathrm{a}$ & $12,65 \mathrm{a}$ & $38,83 \mathrm{a}$ & $31,31 \mathrm{a}$ \\
10 & $7,69 \mathrm{a}$ & $12,62 \mathrm{a}$ & $35,23 \mathrm{a}$ & $3385,39 \mathrm{a}$ \\
11 & $7,49 \mathrm{a}$ & $12,72 \mathrm{a}$ & $34,55 \mathrm{a}$ & $3289,94 \mathrm{a}$ \\
\hline $\mathrm{CV}(\%)$ & $7,69 \mathrm{a}$ & $11,95 \mathrm{a}$ & $34,30 \mathrm{a}$ & 10,08 \\
\hline
\end{tabular}

${ }^{1}$ Médias de tratamentos, seguidas pela mesma letra, não diferem entre si, pelo teste de Tukey, a 5\% de probabilidade. 
autores mencionam que os efeitos do cloreto de mepiquat na cultura do algodoeiro são dependentes de vários fatores, podendo ser destacados: cultivar, época de semeadura, população de plantas, dose e época de aplicação, temperatura, fertilidade do solo e disponibilidade de água para as plantas.

\section{CONCLUSÕES}

1. O cloreto de mepiquat reduz a altura das plantas.

2. A dose de $55 \mathrm{~g}$ de cloreto de mepiquat/ha é suficiente para que as plantas, quando da colheita, tenham altura inferior a $1,30 \mathrm{~m}$.

3. O cloreto de mepiquat reduz o comprimento dos ramos e aumenta a relação entre a matéria seca da parte reprodutiva e vegetativa.

4. As características agronômicas peso de capulho, peso de 100 sementes, porcentagem de fibra e produção de algodão em caroço não são influenciadas significativamente pelo cloreto de mepiquat.

\section{REFERÊNCIAS}

BANZATTO, D.A.; KRONKA, S.N. Experimentação agrícola. 2.ed. Jaboticabal: FUNEP, 1992. 247p.

BARBOSA, L.M.; CASTRO, P.R.C. Desenvolvimento e produtividade de algodoeiros sob efeito de reguladores vegetais. Anais da Escola Superior de Agricultura "Luiz de Queiroz", Piracicaba, v.40, n.1, p.33-86, 1983.

BELTRÃO, N.E. de M.; AZEVÊDO, D.M.P. de. Defasagem entre as produtividades real e potencial do algodoeiro herbáceo: limitações morfológicas, fisiológicas e ambientais. Campina Grande, PB: Embrapa-CNPA, 1993. 108p. (EMBRAPA-CNPA. Documentos, 39)

BUSOLI, A.C.; GRAVENA, S.; SOARES, J.J. Recomendações para MIP-algodão. Jaboticabal: Centro de Manejo Integrado de Pragas. Coordenadoria MIP-Algodão, FCAV/UNESP, 1992. 2p. (Comunicado Técnico).

CARVALHO, L.H.; CHIAVEGATO, E.J.; CIA, E.; KONDO, J.I.; SABINO, J.C.; PETTINELLI JUNIOR, A.; BORTOLETTO, N.; GALLO, P.B. Fitorreguladores de crescimento e capação na cultura algodoeira. Bragantia, Campinas, v.53, n.2, p.247-254, 1994.

CIA, E.; CARVALHO, L.H.; KONDO, J.I.; FUZATTO, M.G.; BORTOLETTO, N.; GALLO, P.B.; CRUZ, L.S.P.; SABINO, N.P.; PTTINELLI JUNIOR, A.;
MARTINS, A.L.M.; SILVEIRA, J.C.D. Efeito do cloreto de clorocolina e cloreto de mepiquat na cultura do algodão (Gossypium hirsutum L.). Planta Daninha, Campinas, v.2, n.7, p.23-36, 1984.

CRUZ, L.S.P.; SABINO, N.P.L.; TOLEDO, N.M.P. Efeitos do cloreto de mepiquat empregado como fitorregulador sobre o algodoeiro herbáceo (Gossypium hirsutum L.). Planta Daninha, Campinas, v.5, n.1, p.15-22, 1982.

FERNANDEZ, C.J.; COTHREN, J.T.; McINNES, K.J. Partitioning of biomass in well-watered and waterstressed cotton plants treated with mepiquat chloride. Crop Science, Madison, v.31, n.5, p.1224-1228, 1991.

HODGES, H.F.; REDDY, V.R.; REDDY, K.R. Mepiquat chloride and temperature effects on photosynthesis and respiration of fruiting cotton. Crop Science, Madison, v.31, n.5, p.1302-1308,1991.

KERBY, T.A. Cotton response to mepiquat chloride. Agronomy Journal, Madison, v.77, n.4, p.515-518, 1985.

MEREDITH JUNIOR, W.R.; WELLS, R. Potential for increasing cotton yields through enhanced partitioning to reproductive structures. Crop Science, Madison, v.29, n.3, p.636-639, 1989.

REDDY, V.R.; BAKER, D.N.; HODGES, H.F. Temperature and mepiquat chloride effects on cotton canopy architecture. Agronomy Journal, Madison, v.82, n.2, p.190-195, 1990.

REDDY, V.R.; TRENT, A.; ACOCK, B. Mepiquat chloride and irrigation versus cotton growth and development. Agronomy Journal, Madison, v.84, n.6, p.930-933, 1992.

SABINO, N.P.; LAZZARINI, J.F.; GRIDI-PAPP, I.L.; FUZATTO, M.G.; GROSSI, J.M.M. Estudo de amostragens de capulhos em canteiros experimentais de algodão. Bragantia, Campinas, v.34, n.8, p.163-169, 1975.

STUART, B.L.; ISBELL, V.R.; WENDT, C.W.; ABERNATY, J.R. Modification of cotton relations and growth with mepiquat chloride. Agronomy Journal, Madison, v.76, n.4, p.651-655, 1984.

WALLACE, T.P.; SNIPES, C.E.; WHITE, B.W. Effects of single-multiple apllications of mepiquat chloride on Mississipi cotton. Research Reports -Mississipi Agricultural Foresty Experiment Station, Mississipi, v.18, n.5, p.5, 1993.

YORK, A.C. Cotton cultivar response to mepiquat chloride. Agronomy Journal, Madison, v.75, n.4, p.663-667, 1983.

Pesq. agropec. bras., Brasília, v.34, n.3, p.369-375, mar. 1999 